\title{
Ground Penetrating Radar Imaging of Buried Metallic Objects
}

\author{
Polat, A. Burak; Meincke, Peter
}

Published in:

Proceedings of IEEE Antennas and Propagation Society International Symposium, Boston, USA

Link to article, DOI:

10.1109/APS.2001.959448

Publication date:

2001

Document Version

Publisher's PDF, also known as Version of record

Link back to DTU Orbit

Citation (APA):

Polat, A. B., \& Meincke, P. (2001). Ground Penetrating Radar Imaging of Buried Metallic Objects. In

Proceedings of IEEE Antennas and Propagation Society International Symposium, Boston, USA (Vol. 4, pp. 264-267) https://doi.org/10.1109/APS.2001.959448

\section{General rights}

Copyright and moral rights for the publications made accessible in the public portal are retained by the authors and/or other copyright owners and it is a condition of accessing publications that users recognise and abide by the legal requirements associated with these rights.

- Users may download and print one copy of any publication from the public portal for the purpose of private study or research.

- You may not further distribute the material or use it for any profit-making activity or commercial gain

- You may freely distribute the URL identifying the publication in the public portal 


\title{
Ground Penetrating Radar Imaging of Buried Metallic Objects
}

Burak Polat and Peter Meincke*1

$\emptyset$ rsted.DTU, Section for Electromagnetic Systems, Build. 348 Technical University of Denmark, DK-2800 Kgs.Lyngby, Denmark

\begin{abstract}
1. Introduction
During the past decade there has been considerable research on ground penetrating radar (GPR) tomography for detecting objects such as pipes, cables, mines, and barrels buried under the surface of the earth. While the earlier researches were all based on the assumption of a homogeneous background for simplicity, the planar air-soil interface has also been taken into account in two recently developed algorithms $[1,2]$. These inversion schemes are both based on the first Born approximation, where a plane wave expansion of the dyadic Green function and an asymptotic formula valid for objects that are buried a few wavelengths from the air-soil interface is introduced in [1], while a far-field expression for the same dyadic Green function, which is valid when the object is located far from the interface, is employed in [2]. Despite the fact that the Born approximation is valid for low contrast scatterers, both inversion schemes have proven successful in detecting the location of high contrast conducting bodies (cf. [1, Fig. 6]).

In this paper we address a general formulation for GPR imaging of buried 3-D metallic objects within the physical optics $(\mathrm{PO})$ approximation which also highlights the analytical background behind the success of methods employed in $[1,2]$ in identifying high contrast scatterers.
\end{abstract}

\section{The Forward Model}

The GPR configuration is shown in Figure 1 in which a planar interface separates air from soil. A Cartesian $x y z$ coordinate system is introduced such that the $x y$ plane coincides with the interface and such that the upper region $z>0$ is air with permittivity $\epsilon_{0}$ and permeability $\mu_{0}$ while the lower region $z<0$ is homogeneous and lossless soil with permittivity $\epsilon_{1}$, permeability $\mu_{0}$. However, the inversion algorithm is still expected to prove successful for small losses in soil as in $[1,2]$ (see $[1,2]$ for details).

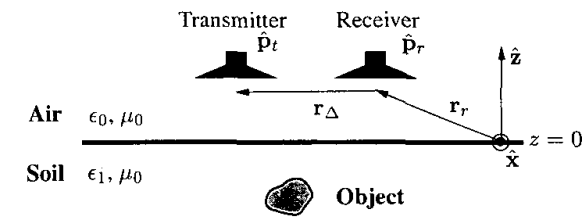

Figure 1: The fixed-offset GPR configuration.

'The Danish Technical Research Council is acknowledged for supporting this work. 
All constitutive parameters are assumed real and constant over the bandwidth of the transmitter. The propagation constant of air is $k_{0}=\omega \sqrt{\mu_{0} \epsilon_{0}}$ and that of soil is $k_{1}=\omega \sqrt{\mu_{0} \epsilon_{1}}$ (time factor $\exp (-i \omega t)$ ). The transmitting and receiving antennas are assumed to be ideal dipoles situated at $\mathbf{r}_{t}=\mathbf{R}_{t}+\hat{\mathbf{z}} z_{t}$ and $\mathbf{r}_{r}=\mathbf{R}_{r}+\hat{\mathbf{z}} z_{r}$ respectively, with the fixed-offset $\mathbf{r}_{\Delta}=\mathbf{r}_{t}-\mathbf{r}_{r}=\mathbf{R}_{\Delta}+\hat{\mathbf{z}} z_{\Delta}$. The transmitting dipole has its dipole moment parallel to the unit vector $\hat{\mathbf{p}}_{t}$, and its current density is denoted by $I(\omega)$. The receiving dipole determines directly the $\hat{\mathrm{p}}_{r}$ component of the electric field.

Replacing the asymptotic expansion of the dyadic Green function $\overline{\mathbf{G}}$ for the planar interface under the far-field approximation (see [2] for details) in the expression of the background electric field [1, eq.(10)]

$$
\mathrm{E}^{b}\left(\mathbf{r}^{\prime}, \omega\right)=i \omega \mu_{0} I(\omega) \hat{\mathbf{p}}_{t} \cdot \overline{\mathbf{G}}\left(\mathbf{r}_{t}, \mathbf{r}^{\prime}, \omega\right), z^{\prime}<0
$$

helps us to express the background electromagnetic field as a uniform plane wave propagating along the $-\hat{\mathbf{R}}_{t o}$ direction as

$$
\mathbf{E}^{b}\left(\mathbf{r}^{\prime}, \omega\right)=\mathbf{E}_{\rho} \exp \left(-i k_{1} \hat{\mathbf{R}}_{t o} \cdot \mathbf{r}^{\prime}\right)
$$

$$
\mathbf{H}^{b}\left(\mathbf{r}^{\prime}, \omega\right)=-\frac{1}{Z_{1}} \hat{\mathbf{R}}_{t o} \times \mathbf{E}_{o} \exp \left(-i k_{1} \hat{\mathbf{R}}_{t o} \cdot \mathbf{r}^{\prime}\right), Z_{1}=\sqrt{\frac{\mu_{0}}{\epsilon_{1}}}
$$

with

$$
\mathbf{E}_{o}=\mathbf{E}_{o}\left(\mathbf{r}_{t}, \mathbf{r}_{o}, \omega\right)=I(\omega) C\left(\mathbf{r}_{t}, \mathbf{r}_{o}, \omega\right) \hat{\mathbf{p}}_{t} \cdot \overrightarrow{\mathbf{F}}\left(\mathbf{K}_{t o}, \omega\right)
$$

$$
\mathbf{E}_{o} \cdot \hat{\mathbf{R}}_{t o}=0
$$

$C\left(\mathbf{r}_{t}, \mathbf{r}_{o}, \omega\right)=i \omega \mu_{0} \gamma_{1}\left(\mathbf{K}_{t o}, \omega\right) \exp \left[i \gamma_{0}\left(\mathbf{K}_{t o}, \omega\right) z_{t}\right] \exp \left[i k_{1}\left(R_{t o}+\hat{\mathbf{R}}_{t o} \cdot \mathbf{r}_{o}\right)\right] \frac{1}{4 \pi R_{t o}}$

where

$$
\mathbf{r}_{o}=\hat{\mathbf{x}} x_{o}+\hat{\mathbf{y}} y_{o}+\hat{\mathbf{z}} z_{o}, z_{o}<0
$$

is the position vector which localizes the buried body and

$$
\begin{gathered}
R_{t o}=\left|\mathbf{R}_{t}-\mathbf{r}_{o}\right|=\sqrt{\left(x_{t}-x_{o}\right)^{2}+\left(y_{t}-y_{o}\right)^{2}+z_{o}^{2}}, \quad \hat{\mathbf{R}}_{t o}=\frac{\mathbf{R}_{t}-\mathbf{r}_{o}}{R_{t o}} \\
\mathbf{K}_{t o}=\hat{\mathbf{x}} k_{1} \frac{x_{t}-x_{o}}{R_{t o}}+\hat{\mathbf{y}} k_{1} \frac{y_{t}-y_{o}}{R_{t o}} \\
\gamma_{0,1}\left(\mathbf{K}_{t o}, \omega\right)=\sqrt{k_{0,1}^{2}-\left|\mathbf{K}_{t o}\right|^{2}}, \operatorname{Re}\left\{\gamma_{0,1}\right\} \geq 0, \operatorname{Im}\left\{\gamma_{0,1}\right\} \geq 0 .
\end{gathered}
$$

In (2c) $\overline{\mathbf{F}}$ stands for a non-invertable dyadic, a closed form expression of which is already available in literature (cf.[1, eq.(6)]). The physical optics current density is expressed as

$$
\begin{array}{r}
\mathbf{J}_{c}^{P O}\left(\mathbf{r}^{\prime}, \omega\right)=2 \boldsymbol{\gamma}_{u}\left(\mathbf{r}^{\prime}\right) \times \mathbf{H}^{b}\left(\mathbf{r}^{\prime}, \omega\right)=-\frac{2}{Z_{1}}\left[\hat{\mathbf{R}}_{t o}\left(\boldsymbol{\gamma}_{u} \cdot \mathbf{E}_{o}\right)-\mathbf{E}_{o}\left(\boldsymbol{\gamma}_{u} \cdot \hat{\mathbf{R}}_{t o}\right)\right] \\
\cdot \exp \left(-i k_{1} \hat{\mathbf{R}}_{t o} \cdot \mathbf{r}^{\prime}\right)
\end{array}
$$


where

$$
\gamma_{u}\left(\mathbf{r}^{\prime}\right)=\gamma\left(\mathbf{r}^{\prime}\right) u\left(\hat{\mathbf{R}}_{t o} \cdot \hat{\mathbf{n}}^{\prime}\right) \hat{\mathbf{n}}^{\prime}
$$

In (3b), $u$ is the unit step function and $\hat{\mathbf{n}}^{\prime}, \gamma$, and $\gamma_{u}$ denote the unit normal vector the scalar singular function [3], and the illuminated side vector singular function of the scatterer surface, respectively.

Replacing (3a) and the asymptotic expression of the dyadic Green function [2] into the integral representation of the scattered field at the receiver point,

$$
\mathbf{E}^{s}\left(\mathbf{r}_{r}, \omega\right)=i \omega \mu_{0} \int_{V} \overline{\mathbf{G}}\left(\mathbf{r}_{r}, \mathbf{r}^{\prime}, \omega\right) \cdot \mathbf{J}_{c}^{P O}\left(\mathbf{r}^{\prime}, \omega\right) d V^{\prime}
$$

yields

$\mathbf{E}^{s}\left(\mathbf{r}_{r}, \omega\right)=-\frac{2}{Z_{1}} C\left(\mathbf{r}_{r}, \mathbf{r}_{o}, \omega\right) \overline{\mathbf{F}}\left(\mathbf{K}_{r o}, \omega\right) \cdot\left[\hat{\mathbf{R}}_{t o}\left(\tilde{\gamma}_{u} \cdot \mathbf{E}_{o}\right)-\mathbf{E}_{o}\left(\tilde{\gamma}_{u} \cdot \hat{\mathbf{R}}_{t o}\right)\right]$

where

$$
\tilde{\gamma}_{u}=\tilde{\gamma}_{u}\left(k_{1}\left(\hat{\mathbf{R}}_{t o}+\hat{\mathbf{R}}_{r o}\right)\right)=\int_{V} \boldsymbol{\gamma}_{u}\left(\mathbf{r}^{\prime}\right) \exp \left[-i k_{1}\left(\hat{\mathbf{R}}_{t o}+\hat{\mathbf{R}}_{r o}\right) \cdot \mathbf{r}^{\prime}\right] d V^{\prime}
$$

Eq. (5a) is a relation between the two projections of the illuminated side vector singular function $\gamma_{u}$ and the expression of the scattered field at the receiver location. Next we shall express $\mathbf{F}$ in (5a) as the multiplication of the transmission dyadic $\overline{\mathbf{T}}_{10}$ for the planar interface and the plane wave spectrum of an arbitrary Hertzian dipole in free space, namely

$$
\overline{\mathbf{F}}\left(\mathbf{K}_{r o}, \omega\right)=\frac{1}{\gamma_{1}\left(\mathbf{K}_{r o}, \omega\right)} \overline{\mathbf{T}}_{10}\left(\mathbf{K}_{r o}, \omega\right) \cdot\left[\overline{\mathbf{I}}-\hat{\mathbf{R}}_{r o} \hat{\mathbf{R}}_{r o}\right] .
$$

We hereby note, without giving its closed-form expression, that $\overline{\mathbf{T}}_{10}$ is invertable and reduces to the identity dyadic $\overline{\mathrm{I}}$ when $k_{0}=k_{1}$.

For the zero-offset case $\hat{\mathbf{R}}_{r o}=\hat{\mathbf{R}}_{t o}$, (2d) yields $\hat{\mathbf{R}}_{r o} \cdot \mathbf{E}_{o}=0$ and the right-hand side of (5a) simplifies as

$\overline{\mathbf{F}}\left(\mathbf{K}_{r o}, \omega\right) \cdot\left[\hat{\mathbf{R}}_{t o}\left(\tilde{\gamma_{u}} \cdot \mathbf{E}_{o}\right)-\mathbf{E}_{o}\left(\tilde{\gamma_{u}} \cdot \hat{\mathbf{R}}_{t o}\right)\right]=\frac{-1}{\gamma_{1}\left(\mathbf{K}_{r o}, \omega\right)} \overline{\mathbf{T}}_{10}\left(\mathbf{K}_{r o}, \omega\right) \cdot \mathbf{E}_{o} \tilde{O}\left(2 k_{1} \hat{\mathbf{R}}_{r o}\right)$

where

$$
O\left(\mathbf{r}^{\prime}\right)=\gamma_{u}\left(\mathbf{r}^{\prime}\right) \cdot \hat{\mathbf{R}}_{t o}
$$

is the scalar object function, and the forward model can be expressed as

$$
\hat{\mathbf{p}}_{r} \cdot \mathbf{E}^{s}\left(\mathbf{r}_{r}, \omega\right)=D\left(\mathbf{r}_{r}, \mathbf{r}_{o}, \omega\right) \tilde{O}\left(2 k_{1} \hat{\mathbf{R}}_{r o}\right)
$$

with

$$
D\left(\mathbf{r}_{r}, \mathbf{r}_{o}, \omega\right)=\frac{2}{Z_{1}} I(\omega) \frac{C^{2}\left(\mathbf{r}_{r}, \mathbf{r}_{o}, \omega\right)}{\gamma_{1}\left(\mathbf{K}_{r o}, \omega\right)} \hat{\mathbf{p}}_{r} \cdot \overline{\mathbf{T}}_{10}\left(\mathbf{K}_{r o}, \omega\right) \cdot\left[\hat{\mathbf{p}}_{r} \cdot \overline{\mathbf{F}}\left(\mathbf{K}_{r o}, \omega\right)\right] .
$$

0-7803-7070-8/01/\$10.00 @2001 IEEE

266 
When compared with the forward model derived under the assumption of the first Born approximation [2], one observes that they, except for an unimportant factor, possess the same form. This explains why the Born-inversion models of $[1,2]$ can be used to detect metallic objects.

The forward model for the fixed-offset case $\hat{\mathbf{R}}_{r o} \neq \hat{\mathbf{R}}_{t o}$ (therefore $\hat{\mathbf{R}}_{r o} \cdot \mathbf{E}_{o} \neq 0$ ), inspired by the frequency diversity algorithms in the works of Langenberg $e t$ al. ( cf. [4]), can be derived by scalar multiplying each side of the relation

$$
\begin{aligned}
\overline{\mathbf{T}}_{10}^{-1}\left(\mathbf{K}_{r o}, \omega\right) \cdot \mathbf{E}^{s}\left(\mathbf{r}_{r}, \omega\right)=-\frac{2}{Z_{1}} \frac{C\left(\mathbf{r}_{r}, \mathbf{r}_{o}, \omega\right)}{\gamma_{1}\left(\mathbf{K}_{r o}, \omega\right)}\left[\overline{\mathbf{I}}-\hat{\mathbf{R}}_{r o} \hat{\mathbf{R}}_{r o}\right] \\
\cdot\left[\hat{\mathbf{R}}_{t o}\left(\overline{\gamma_{u}} \cdot \mathbf{E}_{o}\right)-\mathbf{E}_{o}\left(\tilde{\gamma_{u}} \cdot \hat{\mathbf{R}}_{t o}\right)\right]
\end{aligned}
$$

by $\hat{\mathbf{R}}_{t o}$ and $\mathbf{E}_{o}^{*}$ respectively to get two equations for the two projections $\tilde{\gamma}_{u} \cdot \mathbf{E}_{o}$ and $\tilde{\gamma}_{u} \cdot \hat{\mathbf{R}}_{t o}$ as follows:

$\left[\begin{array}{c}\hat{\mathbf{R}}_{t o} \cdot \overline{\mathbf{T}}_{10}^{-1} \cdot \mathbf{E}^{s} \\ \mathbf{E}_{o}^{*} \cdot \overline{\mathbf{T}}_{10}^{-1} \cdot \mathbf{E}^{s}\end{array}\right]=-\frac{2}{Z_{1}} \frac{C\left(\mathbf{r}_{r}, \mathbf{r}_{o}, \omega\right)}{\gamma_{1}\left(\mathbf{K}_{r o}, \omega\right)}$

$$
\cdot\left[\begin{array}{cc}
1-\left(\hat{\mathbf{R}}_{t o} \cdot \hat{\mathbf{R}}_{r o}\right)^{2} & \left(\hat{\mathbf{R}}_{t o} \cdot \hat{\mathbf{R}}_{r o}\right)\left(\hat{\mathbf{R}}_{r o} \cdot \mathbf{E}_{o}\right) \\
-\left(\hat{\mathbf{R}}_{t o} \cdot \hat{\mathbf{R}}_{r o}\right)\left(\hat{\mathbf{R}}_{r o} \cdot \mathbf{E}_{o}^{*}\right) & \left|\hat{\mathbf{R}}_{r o} \cdot \mathbf{E}_{o}\right|^{2}-\left|\mathbf{E}_{o}\right|^{2}
\end{array}\right]\left[\begin{array}{c}
\tilde{\gamma}_{u} \cdot \mathbf{E}_{o} \\
\tilde{\gamma}_{u} \cdot \hat{\mathbf{R}}_{t o}
\end{array}\right]
$$

Here the asterix denotes the complex conjugate and the presence of the inverse of this matrix is obvious since its determinant $\left|\hat{\mathbf{R}}_{r o} \cdot \mathbf{E}_{o}\right|^{2}+\left|\mathbf{E}_{o}\right|^{2}\left(\left(\hat{\mathbf{R}}_{t o} \cdot \hat{\mathbf{R}}_{r o}\right)^{2}-1\right)$ is identically nonzero.

\section{Concluding Remarks}

We hereby like to note that in practice it is not necessary to measure the $z-$ component of the scattered electric field for fixed-offset case; since the $z-$ components of $\mathbf{E}_{o}$ (due to eq. (2d)) and $\hat{\mathbf{R}}_{t \omega}, \hat{\mathbf{R}}_{r o}$ can be expressed as a linear combination of their other two components, one can always get sufficient information by extracting the first two components of these vectors and the first two rows and columns of the transmission dyadic $\overline{\mathrm{T}}_{10}$.

\section{References}

[1] T.B. Hansen and P. Meincke Johansen, "Inversion scheme for ground penetrating radar that takes into account the planar air-soil interface." IEEE Trans. Geosci. Remote Sensing vol. 38, no. 1, Jan. 2000.

[2] P. Meincke, "2.5-D Far-field diffraction tomography inversion scheme for GPR that takes into account the planar air-soil interface", submitted to IEEE AP-S International Symposium, Boston, MA, July 8-13, 2001

[3] N. Bleistein, Mathematical methods for wave phenomena, Academic Press, Orlando 1983.

[4] K.J. Langenberg, M. Brandfass, P. Fellinger, T. Gurke, and T. Kreutter, "A unified theory of multidimensional electromagnetic vector inverse scattering within the Kirchoff or Born approximation." In H. Überall and W.M. Boerner (Eds.) Radar target Imaging, Springer-Verlag, Berlin, 1994. 